\title{
Study on the Optoelectronic Design for Korean Mobile Satellite Laser Ranging System
}

\author{
Hyung-Chul Lim ${ }^{\dagger}$, Seong-Cheol Bang, Sung-Yeol Yu, Yoon-Kyung Seo, Eunseo Park, \\ Kwang Dong Kim, Jakyoung Nah, Jeong Gyun Jang, Bi-Ho Jang, Jang-Hyun Park, and \\ Jong-Uk Park
}

Korea Astronomy and Space Science Institute, Daejeon 305-348, Korea

Korea Astronomy and Space Science Institute has been developing one mobile and one stationary satellite laser ranging system for the space geodesy research and precise orbit determination since 2008, which are called as ARGO-M and ARGO-F, respectively. They will be capable of daytime laser ranging as well as nighttime and provide the accurate range measurements with millimeter level precision. Laser ranging accuracy is mostly dependent on the optics and optoelectronic system which consists of event timer, optoelectronic controller and photon detectors in the case of ARGO-M. In this study, the optoelectronic system of ARGO-M is addressed and its critical design is also presented. Additionally, the experiment of the integrated optoelectronic system was performed in the laboratory to validate the functional operation of each component and its results are analyzed to investigate ARGO-M performance in advance.

Keywords: ARGO-M, satellite laser ranging, space geodesy, optoelectronic system

\section{INTRODUCTION}

Satellite laser ranging (SLR) is the most accurate satellite ranging technique using an ultra-shot pulse laser for space geodesy, geophysics and precise orbit determination. The technique measures the round trip time of photons to satellites equipped with the laser retro-reflector array (LRA), which provides the range measurements with $\mathrm{mm}$ level precision of normal point (NP) data. Recent SLR technology shows four trends to reduce operation cost and improve the quantity of measurement data: high level of automation, kilohertz laser ranging, eyesafe operation and millimeter accuracy (Noll \& Pearlman 2009). In particular, many SLR stations have tried to upgrade toward a kilohertz system using ultra-shot laser pulse or a fully automated system. The kilohertz system was tried by Graz station in 2003 at first time to increase the quantity of measurement data, which are now oper- ated by several stations: Graz (Austria), NGSLR (USA), Herstmonceux (England) and some stations in China.

The SLR system consists of optics, optoelectronics, laser, tracking mount and operation software. All of them have influence on system performance such as link budget, daylight tracking capability and measurement precision (Lim et al. 2010). The optoelectronic system plays an important role in measuring the start and stop epochs of laser pulses and also in coltrolling some devices for the accurate range measurement. So the system consisting of photon detectors, event timing system and optoelectronic controller is the crucial factor that affects the ranging accuracy of SLR system directly.

Korea Astronomy and Space Science Institute (KASI) has been developing one mobile and one stationary SLR system for the space geodesy research and precise orbit determination since 2008, which are called as ARGO-M and ARGO-F, respectively. ARGO-M, which will be com-

(c) This is an Open Access article distributed under the terms of the Creative Commons Attribution Non-Commercial License (http://creativecommons.org/licenses/by-nc/3.0/) which permits unrestricted non-commercial use, distribution, and reproduction in any medium, provided the original work is properly cited.

Received Oct 10,2010 Revised May 29, 2011 Accepted June 08, 2011 ${ }^{\dagger}$ Corresponding Author

E-mail: hclim@kasi.re.kr

Tel: +82-42-865-3235 Fax: +82-42-861-5610 
pletely developed in 2011, has the separate optical path that employs the $40 \mathrm{~cm}$ receiving and $10 \mathrm{~cm}$ transmitting telescopes. It is a remotely controllable and $\mathrm{kHz}$ laser ranging system with the single shot range precision less than one centimeter and NP precision better than 5 $\mathrm{mm}$ for laser geodynamics satellite (LAGEOS). ARGO-M will be capable of tracking satellites with LRA up to the altitude of 25,000 km and provides 24 hour tracking coverage including daylight tracking. ARGO-M uses a diode pumped laser with $532 \mathrm{~nm}$ wavelength, the original output energy of $2.8 \mathrm{~mJ}$ at $2 \mathrm{kHz}, 15 \mathrm{ps}$ pulse width. In contrast, ARGO-F has the common coude optical path using the telescope of $100 \mathrm{~cm}$ aperture, whose requirements are not fixed yet because its development is planned to be started from 2012 and finished in 2014.

In this study, the critical design is addressed for the optoelectronic system of ARGO-M as well as its configuration. The characteristics and specifications of the optoelectronic system are analyzed based on the component level to achieve the required performance of ARGO-M which has NP precision less than $5 \mathrm{~mm}$ for LAGEOS satellite. To validate the performance of the optoelectronic system, pulse distribution unit (PDU) developed by KASI is analyzed in terms of system delay. In addition, the experiment of the integrated optoelectronic system was performed by using the laser system, ground target and receiving optics in the laboratory to investigate if each component works well and to analyze the laser ranging precision.

\section{OPTOELECTRONIC SYSTEM OF ARGO-M}

As shown in Fig. 1, the optoelectronic system of ARGO-M consists of three components: event timer, optoelectronic controller and transmitting/receiving photon detectors. The photon detectors consist of start pulse detector (SPD), compensated single photon avalanche diode (C-SPAD) and PDU. The SPD, which is installed behind the second reflecting mirror on the transmitting optical table, detects the leakage laser pulses through the reflecting mirror and then converts them to electrical signals. The C-SPAD in the focal plane of the receiving telescope also detects returned photons from satellites within the signal strength ranging from a single photon to hundreds of photons. Both electrical signals from SPD and C-SPAD are transferred to the event timer after transformed into appropriate types by PDU for their standard interfaces. The event timer measures the event epochs of start signals and stop signals accurately based on 1 pulse per second (PPS) sync pulses and $10 \mathrm{MHz}$ reference frequency from the global positioning system (GPS) time frequency receiver. The optoelectronic controller, which is implemented by field programmable gate array (FPGA) board for the fast functional operation, generates range gate (RG) and $2 \mathrm{kHz}$ commands for laser fires by using 1 PPS pulses and $10 \mathrm{MHz}$ reference frequency. The event timer and the optoelectronic controller have interfaces with the operation system for event epochs, RG and device control.

ARGO-M is designed to be capable of tracking satellites in the altitude ranging from $300 \mathrm{~km}$ to $25,000 \mathrm{~km}$ with the $\mathrm{kHz}$ laser system and supply the system performance within $5 \mathrm{~mm}$ of NP precision for LAGEOS satellite. To fulfill these requirements, the optoelectronic system requires the fast RG generation, real-time operation, high quantum efficiency of the receiving detector and laser overlap avoidance function to minimize noises from the backscattering. Table 1 shows the specifications of the optoelectronic system components for these requirements.

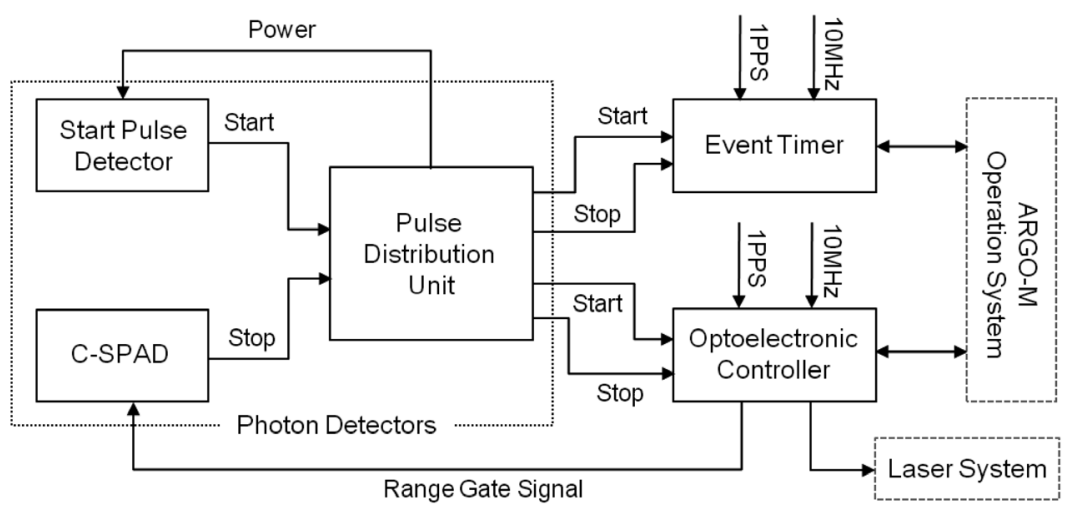

Fig. 1. Configuration of ARGO-M optoelectronic system. C-SPAD: compensated single photon avalanche diode. 
Table 1. Specification analysis for requirements of the optoelectronic system.

\begin{tabular}{|c|c|c|c|c|}
\hline \multicolumn{2}{|c|}{ Item } & Requirement & Component & Specification \\
\hline \multicolumn{2}{|c|}{ Quantum efficiency (C-SPAD) } & $\geq 20 \%$ & C-SPAD & $\geq 20 \%$ \\
\hline \multicolumn{2}{|c|}{$\mathrm{kHz}$ laser ranging } & $\geq 2 \mathrm{kHz}$ & $\begin{array}{l}\text { Event timer } \\
\text { FPGA board }\end{array}$ & $\begin{array}{l}\leq 10 \mathrm{kHz} \\
\geq 2 \mathrm{kHz}\end{array}$ \\
\hline Fast RG & Precision & $\leq 5 \mathrm{~ns}$ & FPGA board & $\leq 1 \mathrm{~ns}$ \\
\hline Generation & Resolution & $\leq 2 \mathrm{~ns}$ & & $0.5 \mathrm{~ns}$ \\
\hline \multicolumn{2}{|c|}{ Time synchronization } & $\mathrm{O}$ & Event timer & $\mathrm{O}$ \\
\hline \multicolumn{2}{|c|}{ Laser overlap avoidance } & $\mathrm{O}$ & FPGA board & $\mathrm{O}$ \\
\hline \multicolumn{2}{|c|}{ Epoch time NP data } & $\leq 0.1 \mu \mathrm{s}$ & Event timer & $\leq 100 \mathrm{~ns} / \mathrm{s}$ \\
\hline
\end{tabular}

C-SPAD: compensated single photon avalanche diode, RG: range gate, FPGA: field programmable gate array, NP: normal point.

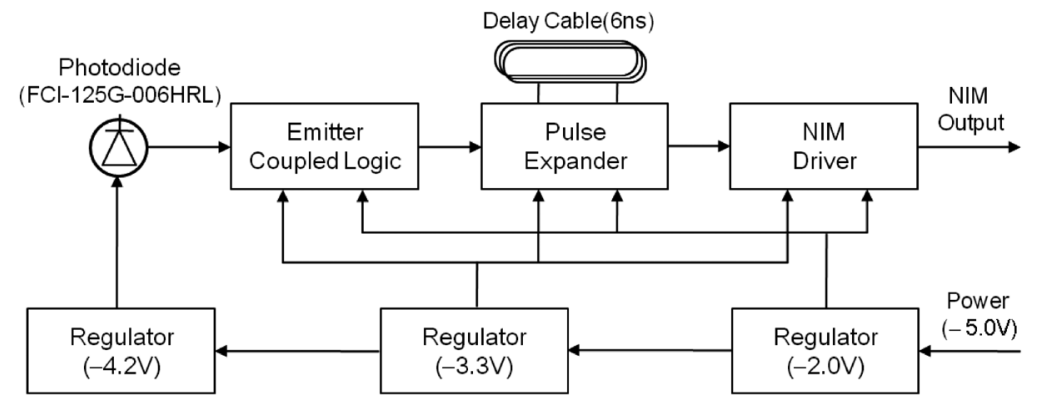

Fig. 2. Block diagram of start pulse detector.

\subsection{Photon Detectors}

The SPD made by KASI consists of a photodiode, emitter coupled logic, pulse expander including delay cable, nuclear instrumentation module (NIM) driver and regulators as shown in Fig. 2. FCI-125G-006HRL (OSI optoelectronics, Chadron Ave Hawthorne, CA, USA) was selected as the photodiode because it has good response performance such as the rising time of $38 \mathrm{ps}$ and the falling time of 313 ps. The pulse width of signals from the emitter coupled logic is so $1 \mathrm{~ns}$ that the event timer and the optoelectronic controller do not detect these signals through PDU. To solve this problem, the pulse expander makes the pulse width to be 6 ns using delay cable, which consists of D-type flip-flop and semi-rigid cable with 1 $\mathrm{mm}$ thickness. The expanded signals are changed to NIM interface signals by NIM driver and then transferred into PDU. One linear regulator supplies the photodiode with $-4.2 \mathrm{~V}$ power and two other linear regulators dispense -3.3 $\mathrm{V}$ and $-2.0 \mathrm{~V}$ power to the emitter coupled logic, the pulse expander and the NIM driver.

The SPAD detector has some advantages over the traditional photo-multiplier tubes (PMTs): the fast rise time of the avalanche and high quantum efficiency. But it has significant dead time from 50 to 100 ns, and intrinsic noise called as time-walk error that the response time is dependent on both photon energy and the temperature of the device. The C-SPAD used in ARGO-M has the time walk compensation circuit and the temperature control unit to maintain $-60^{\circ} \mathrm{C}$ (Kirchner et al. 1998), whose specifications are shown in Table 2.

Table 2. Specifications of C-SPAD.

\begin{tabular}{|c|c|}
\hline Item & Specifications \\
\hline Operating principle & $\begin{array}{l}\text { Si avalanche photodiode pulse biased } \\
\text { above break }\end{array}$ \\
\hline Quantum efficiency & >20\%@532nm \\
\hline Timing resolution & 48 ps full width half maximum \\
\hline Timing delay stability & $\sim 1$ ps \\
\hline Dark count & $\begin{array}{l}\text { Rate }<10 \text { kHz@200 } \mu \mathrm{m} \text { chip, }<2 \text { kHz @ } \\
100 \mu \mathrm{m} \text { chip }\end{array}$ \\
\hline Mode of operation & Gated \\
\hline Gate width & $1 \mathrm{~ns} \sim 100 \mathrm{us}$ \\
\hline Gate on risetime & $100 \mathrm{ps} \sim 2 \mathrm{~ns}$ \\
\hline Gate on duty cycle & No limit \\
\hline Output & NIM like, 0/-0.9 V, 50 Ohms \\
\hline Gate & Pulse TTL levels, 50 Ohms \\
\hline Damage threshold & $1 \mathrm{~nJ} @ 1 \mathrm{~ns}$ pulse \\
\hline Input optics & $\begin{array}{l}\text { Optional, } \mathrm{f}=12 \mathrm{~mm}, \mathrm{f} / \mathrm{D}=1 \text {, aluminum- } \\
\text { coated }\end{array}$ \\
\hline
\end{tabular}

C-SPAD: compensated single photon avalanche diode, NIM: nuclear instrumentation module, TTL: transistor-transistor logic. 
The PDU distributes two signal types for the event timer, the optoelectronic controller and various experimental purposes. One is NIM type signal and the other is transistor-transistor logic (TTL) type. As shown in Fig. 3 , it has one pair of input buffers and four pairs of output signals made by two NIM drivers and TTL drivers. Both start signal from SPD and stop signal from C-SPAD go into the input buffers and then are converted to NIM signals for the event timer and to TTL signals for the optoelectronic controller. Other two pairs of NIM and TTL signals are used for the experimental purpose. The propagation delay of PDU device means the difference time between when input signal comes in and goes out through signal processing in the device. In the case of ARGO-M PDU device, the value of propagation delay is $250 \mathrm{ps}$, and jitter and propagation delay dispersion is about $50 \mathrm{ps}$.

\subsection{Event Timer}

The event timing system measures the time of flight

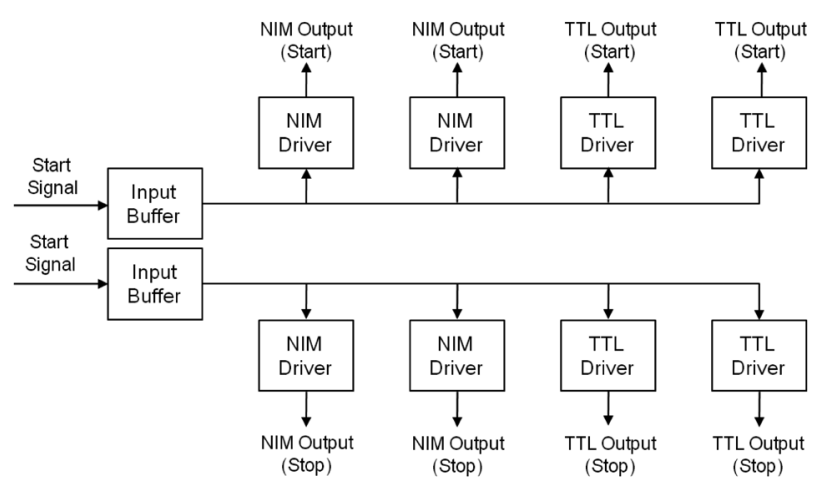

Fig. 3. Block diagram of pulse distribution unit. NIM: nuclear instrumentation module, TTL: transistor-transistor logic.

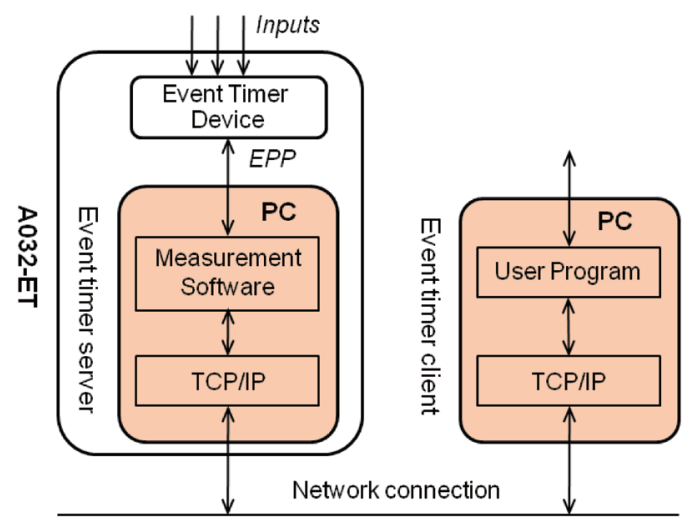

Fig. 4. Client-server architecture of A032-ET (Bespalko et al. 2006).
(TOF) of photons between station and satellites, which is synchronized with GPS time. There are two kinds of the event timing systems: the time interval counter and the event timer. Different from the time interval counter, the event timer records the epochs of start and stop signals and puts them into buffer so that $\mathrm{kHz}$ laser ranging is implemented. ARGO-M uses A032-ET model (Bespalko et al. 2006) as the event timer with root mean square (RMS) resolution less than 10 ps and dead time of 60 ns by using the $100 \mathrm{MHz}$ internal clocks. The A032-ET consists of the event timer device and its controller with the clientserver architecture as shown in Fig. 4. The communication between the event timer device and the event timer server is established by enhanced parallel port with maximum transmission speed of $8 \mathrm{Mbps}$.

The A032-ET executes the event measurement with two stages. In the first stage, it transforms every event into a single 80-bit timing data block and then accumulates it in a first-in, first-out (FIFO) memory. The timing data block contains 39 bits of the counting data with $10 \mathrm{~ns}$ resolution and 40 bits of the interpolating data as well as one-bit mark specifying start or stop event. At the next stage, the event timer server brings timing data blocks from the FIFO memory and then processes them to obtain the corresponding epoch time-tags in a unified form. In the processing of timing data blocks, the actual physical characteristics of time interpolation is taken into account to obtain the best precision through hardware calibration before measurement. Table 3 shows the specifications of A032-ET.

\subsection{Optoelectronic Controller}

ARGO-M will have $\mathrm{kHz}$ laser ranging capability which requires a fast optoelectronic control of RG generation and laser fire command. So ARGO-M uses the FPGA board with industry standard architecture interface for this performance, which was developed by Graz station in Austria and consists of internal event timer, RG generator and laser fire controller.

The internal event timer determines laser firing and returning epochs independently to compute TOF, which requires several hundred microseconds to several milliseconds to fix the event time. In the case of low-earth-orbit satellites, this time is so long that RG generation of the detector is open after return signals arrive at the detector. To solve this problem, the Graz FPGA board includes four internal event timers with fast response time and resolution of $5 \mathrm{~ns}$ and 500 ps. The fast response digital event timer is implemented by a $200 \mathrm{MHz}$ counter and a ver- 
Table 3. Specifications of A032-ET.

\begin{tabular}{|c|c|c|}
\hline \multicolumn{2}{|c|}{ Item } & \multirow{2}{*}{$\begin{array}{c}\text { Specifications } \\
\text { NIM pulse (falling edge; }>5 \text { ns width) }\end{array}$} \\
\hline Inputs (BNC) & INPUT A & \\
\hline & INPUT B & NIM pulse (falling edge; >5 ns width) \\
\hline & GATE IN & NIM pulse (high level) \\
\hline & SYNC IN & TTL pulse (rising edge, 1 PPS) \\
\hline & TRIG IN & TTL pulse (rising edge) \\
\hline & TRIG IN & $10 \mathrm{MHz}(>0.5 \mathrm{~V}$ p-p) \\
\hline Outputs (BNC) & TEST OUT & NIM pulse indicating the gate delay (option) \\
\hline \multicolumn{2}{|c|}{ Single-shot RMS resolution } & $<10 \mathrm{ps}$ \\
\hline \multicolumn{2}{|l|}{ Dead time } & $60 \mathrm{~ns}$ \\
\hline \multicolumn{2}{|c|}{ Non-linearity error } & $<1$ ps (<3 5 ps for time intervals less than $100 \mathrm{~ns})$ \\
\hline \multicolumn{2}{|c|}{ Offset temperature stability } & $<0.5 \mathrm{ps} /{ }^{\circ} \mathrm{C}$ after warm-up \\
\hline \multicolumn{2}{|l|}{ Warm-up time } & 2 hours \\
\hline \multicolumn{2}{|l|}{ FIFO depth } & 12,000 time-tags \\
\hline \multirow{2}{*}{\multicolumn{2}{|c|}{ Measurement rate }} & Up to $10 \mathrm{kHz}$ mean rate continuously \\
\hline & & Up to $500 \mathrm{~Hz}$ cycle repetition rate \\
\hline \multicolumn{2}{|l|}{ Triggering } & External or internal (programmable) \\
\hline \multicolumn{2}{|l|}{ Time-base } & External or internal $(+/-10 \mathrm{ppm})$ for optional use \\
\hline \multicolumn{2}{|c|}{ Stop-pulse gating } & Only external \\
\hline \multicolumn{2}{|l|}{ Control } & Remote control from a user program via the network \\
\hline
\end{tabular}

nier for the intervals of $500 \mathrm{ps}$. After detecting the event time of start pulse in the internal event timer of the FPGA board, the expected event time of return pulse is calculated within the FPGA board using RG information from the operation system. And the expected time is loaded into the RG generator which activates C-SPAD gated about $65 \mathrm{~ns}$ before the expected arrival of the return photons. The RG generator is also implemented within the FPGA board with full digital type by using a $200 \mathrm{MHz}$ clock and a chain of AND gates, which gives a resolution of 500 ps and a precision less than $1 \mathrm{~ns}$ (Iqbal et al. 2008).

The FPGA board has also the laser fire controller which generates laser fire triggers ranging from $10 \mathrm{~Hz}$ to $2 \mathrm{kHz}$. In the case of $\mathrm{kHz}$ laser ranging to satellites, there exist repeated periods of overlaps within dozens of microseconds between returned photons and just fired laser shots. The backscatter caused by these overlaps would cause significant noise on C-SPAD, which leads to a decrease in the detection probability of the returned photon. To avoid this problem, the laser fire controller generates all the laser firing and laser control commands. Because the expected arrival time of returned photon is already known in the FPGA board, the laser fire controller delays the laser firing command to about $50 \mu$ s if photons are expected to return within $30 \mu \mathrm{s}$.

Besides the three main functions of event timing, RG generation and laser firing command, the FPGA board supplies many interfaces for the tracking mount, the observer cabin, the laser system and optical device in- cluding mirrors and switches. Fig. 5 shows the functional block diagram of the Graz FPGA board used in ARGO-M. The Graz FPGA board mainly consists of internal event timer, frequency dividing block and internal timer, laser controller and RG generator module which also have the function of RG generation for PMT as well as SPAD.

\section{TEST AND RESULTS OF OPTOELECTRONIC SYSTEM}

The SPD and PDU among the optoelectronic components were developed by KASI, while the others have been purchased from the foreign institutes or company. To guarantee and validate the performance of PDU, it is tested and analyzed in terms of system delay. DG645 (Stanford Research Systems, Sunnyvale, CA, USA) was used as a digital delay generator, while A032-ET was used to measure time intervals between start and stop pulses, for the evaluation of PDU performance. The start and stop pulses are generated by DG645 and then transferred to A032-ET via PDU and finally time intervals are derived by A032-ET. The performance test of PDU was done to analyze the difference of time intervals when the start and stop pulses are transmitted to A032-ET through PDU or directly without PDU. In this experiment, real time interval generated by DG645 changes from 200 ns to $210 \mathrm{~ns}$ with $1 \mathrm{kHz}$ repetition rate. Fig. 6 shows each RMS value of time intervals measured by A032-ET for 500 seconds. The 


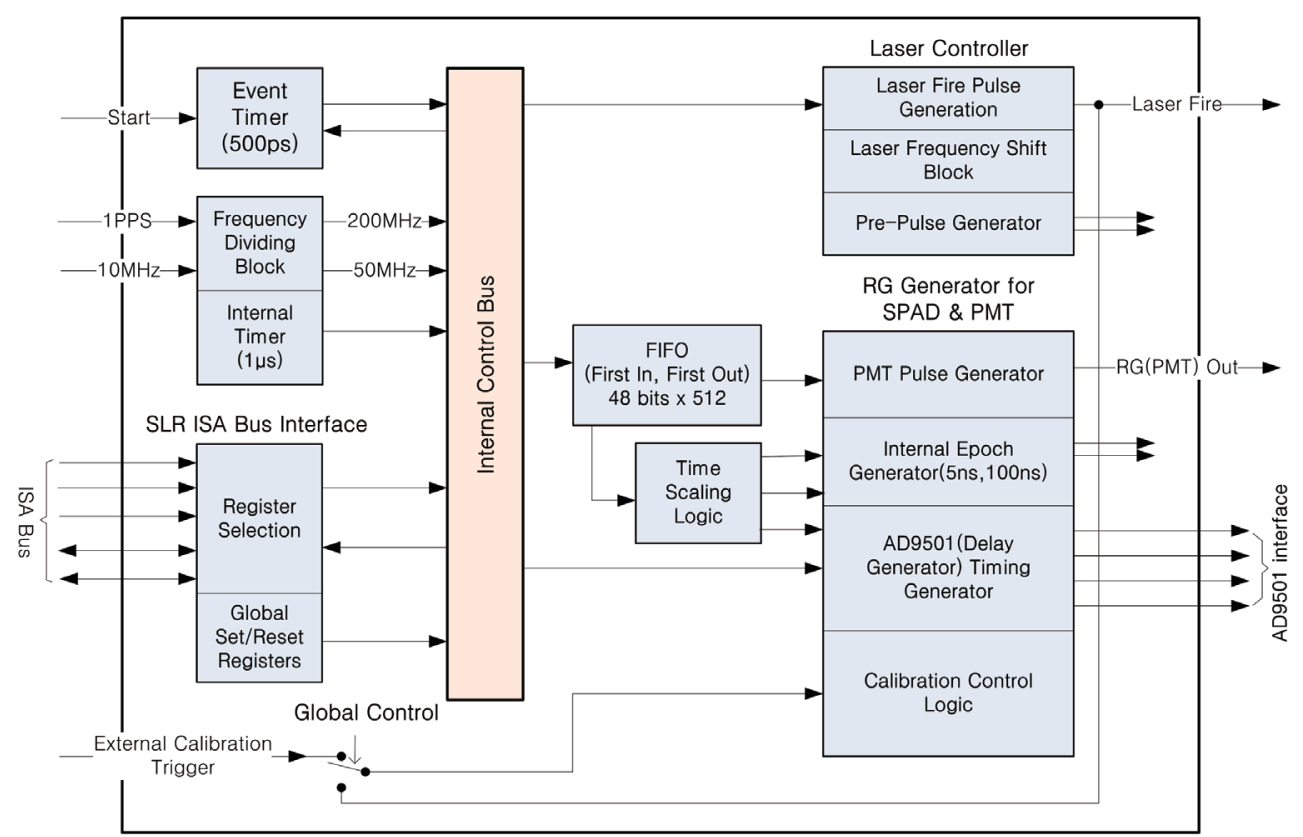

Fig. 5. Functional block diagram of the Graz field programmable gate array board. FIFO: first-in, first-out, ISA: industry standard architecture, PMT: photo-multiplier tube, RG: range gate, SLR: satellite laser ranging, SPAD: single photon avalanche diode.

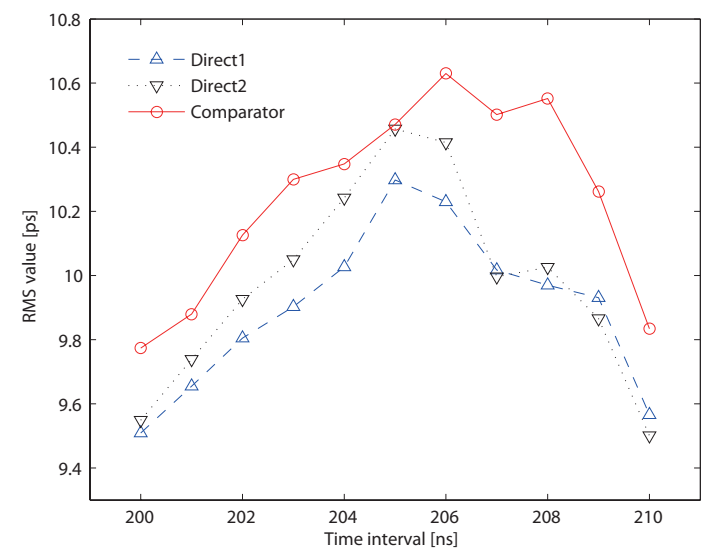

Fig. 6. Performance experiment of pulse distribution unit. RMS: root mean square.

solid line (indicated as comparator in Fig. 6) means RMS values of time intervals when the start and stop pulses are transferred to the A032-ET via PDU. Both the dashed line and dotted line, indicated as Directl and Direct2 respectively, represent RMS values of time intervals when the start and stop pulses are transmitted to the A032ET directly. But the experiments of Direct 1 and Direct 2 were done under the same environment. In the cases of Direct1 and Direct2, RMS values of time intervals vary within 1 ps due to the jitter of DG645. Compared with Directl and Direct2 cases, the PDU shows RMS difference within $0.4 \mathrm{ps}$, which means it functions properly because NP precision of general SLR system is less than 15 ps for LAGEOS satellite.

The experiment of the integrated optoelectronic system was performed with $2 \mathrm{kHz}$ repetition rate on the optical table in the laboratory to validate the performance of the optoelectronic system. As shown in Fig. 7, it has the block diagram similar to the ground calibration or ground laser ranging to correct the system error. In general, the optoelectronic controller generates RG after it transmits the laser fire command and then the start pulse is arrived to the event timer through PDU and it receives the start epoch. However, the FPGA board of the optoelectronic controller generates RG and the laser fire command simultaneously in this experiment because the system delay is so large that the C-SPAD does not detect the stop pulse if the FPGA board receives the start epoch from the internal event timer and then generates RG.

The laser system used in the experiment has high power and ultra-short pulse as shown in Table 4, which is the same as RGL-532 (Photonics Industries, Bohemia, NY, USA) in ARGO-M. Two beam expanders were used to expand the laser beam to $28.5 \mathrm{~mm}$ because the beam diameter at the laser head exit is about $1.9 \mathrm{~mm}$. The ground target consists of a prism, a diffuser, an iris and several neutral density filters. The prism is aluminum-coated for $532 \mathrm{~nm}$ to reduce the reflected energy $0.1 \%$, which has an 


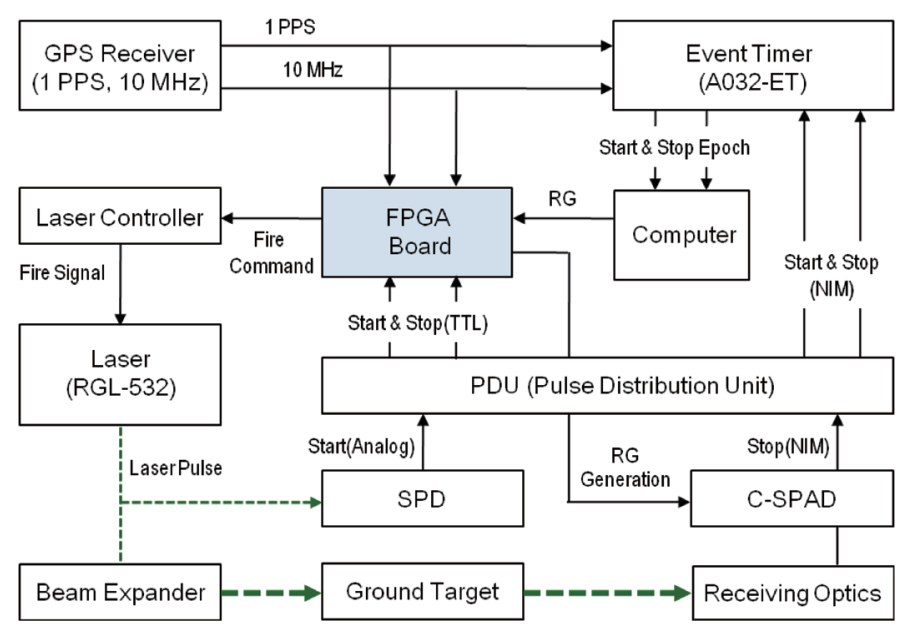

Fig. 7. Block diagram for the experiment of the integrated optoelectronic system. C-SPAD: compensated single photon avalanche diode, GPS: global positioning system, FPGA: field programmable gate array, NIM: nuclear instrumentation module, PPS: pulse per second, SPD: start pulse detector, TTL: transistor-transistor logic.

Table 4. Specifications of the laser system.

\begin{tabular}{ll}
\hline \multicolumn{1}{c}{ Item } & Specifications \\
\hline Wavelength & $532 \mathrm{~nm}$ \\
Pulse energy & $2.8 \mathrm{~mJ} @ 2 \mathrm{kHz}$ \\
Pulse width & $15 \mathrm{ps}$ \\
Repetition rate & $1 \mathrm{~Hz} \sim 10 \mathrm{kHz}$ \\
Pointing stability (full-angle) & $<20 \mu \mathrm{rad}$ \\
Full-angle far-field divergence & $0.5 \mathrm{mrad}$ \\
Beam quality factor $\left(\mathrm{M}^{2}\right)$ & 1.21 \\
\hline
\end{tabular}

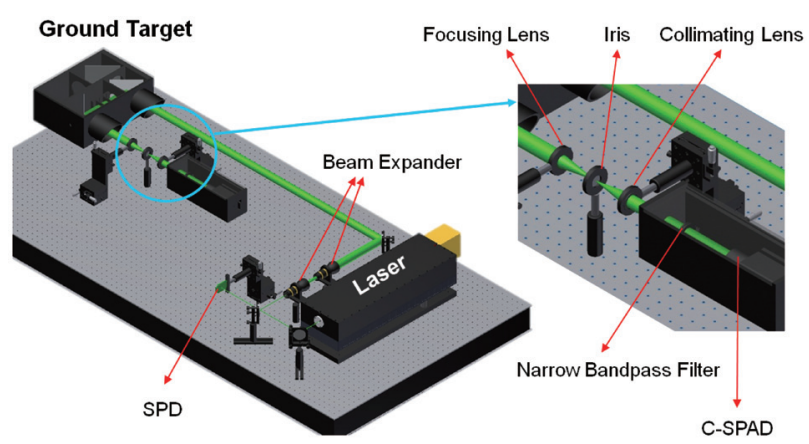

Fig. 8. Optical configuration for the experiment of the integrated optoelectronic system. C-SPAD: compensated single photon avalanche diode, SPD: start pulse detector.

advantage to avoid any secondary reflection (Kirchner \& Koidl 1996). The diffuser is a black anodized aluminum plate with $3 \%$ reflectivity to attenuate the laser power as neutral density filters do. As shown in Fig. 8, the receiving optics consists of a focusing lens, a collimating lens and an iris with $100 \mu \mathrm{m}$ pinhole. The iris is located at the focal plane to remove noises and the collimating lens is used
Table 5. Results of the integrated optoelectronic system.

\begin{tabular}{lccrc}
\hline \multicolumn{1}{c}{ Case (power) } & $\begin{array}{c}\text { Number of } \\
\text { measurements }\end{array}$ & RMS (ps) & Skewness & Kurtosis \\
\hline Case1 $(40 \mathrm{~mW})$ & 33,152 & 46.0 & 0.064 & 2.368 \\
Case2 $(115 \mathrm{~mW})$ & 42,825 & 31.7 & -0.133 & 2.414 \\
Case3 $(320 \mathrm{~mW})$ & 42,783 & 20.7 & -0.061 & 2.365 \\
Case4 $(570 \mathrm{~mW})$ & 43,638 & 14.6 & -0.062 & 2.331 \\
Case5 $(1,090 \mathrm{~mW})$ & 43,687 & 11.3 & 0.084 & 2.321 \\
\hline
\end{tabular}

RMS: root mean square.

to make parallel rays of light. In addition, a narrow bandpass filter is in the front of C-SPAD to pass wavelengths within $532 \pm 1.0 \mathrm{~nm}$.

The experiment was performed under the same circumstance with varying the laser power which has influence on laser ranging accuracy. Table 5 shows the experiment results after data reduction of post-processing based on statistics. For all cases, the laser shot for 25 seconds which make 500,000 measurements because the repetition rate is $2 \mathrm{kHz}$. As the laser power increases, the return rate grows and RMS of laser ranging gets better. But the values of skewness and kurtosis seem to be independent on the laser power. Graz station, as one of the SLR stations with the best performance, has about 16 ps RMS for the ground calibration (Kirchner 2011). The experiment results show that ARGO-M can be operated by $2 \mathrm{kHz}$ repetition rate and its ranging accuracy can reach dozens of picoseconds. However, the suitable laser power should be selected through the tuning process after the system integration and alignment of ARGO-M because the actual return rate of satellites is very low and so the 
ground calibration cannot correct the system error accurately in the case of its high return rate.

Figs. 9 and 10 show the range measurements before the post-processing and histogram after the post-processing for Case 3. As shown in Fig. 9, real measurements stay in the solid line in the vicinity of about $156 \mathrm{~ns}$ but noises are distributed uniformly. The histogram, appeared in Fig. 10, means how many measurements distribute in the neighborhood of the mean value after post-processing. All real measurements have symmetrical and Gaussian distribution with -0.061 value of skewness and 2.365 value of kurtosis in Fig. 10.

\section{CONCLUSIONS}

ARGO-M is the first Korean SLR system, which has been developed by KASI since 2008 for the space geodesy research. The optoelectronic system of ARGO-M is designed to have the NP precision within $5 \mathrm{~mm}$ for LAGEOS satellite. In this study, the critical design of the op-

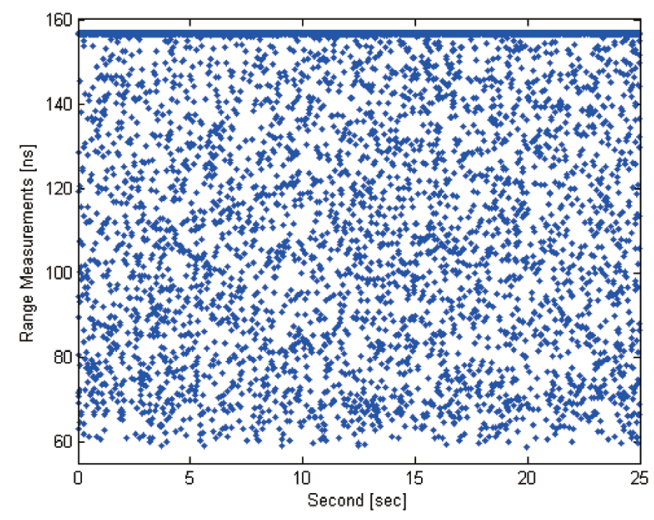

Fig. 9. Range measurements of Case3 before the post-processing.

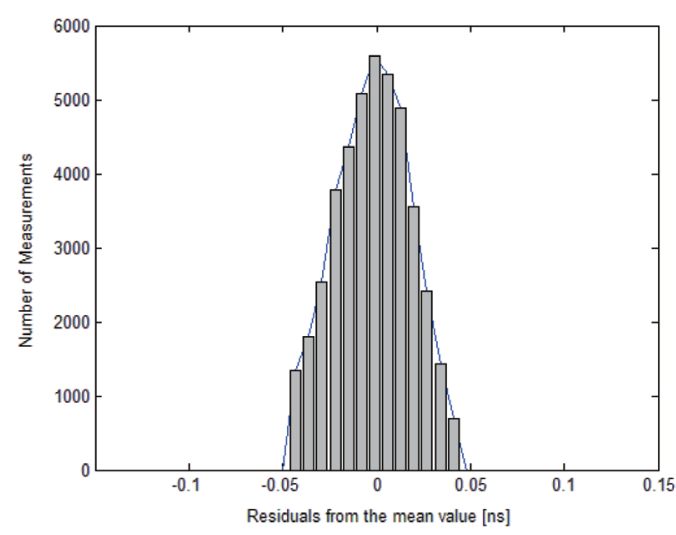

Fig. 10. Histogram of Case3 after the post-processing. toelectronic system is addressed and the characteristics and specifications of its components are also analyzed. Additionally, the experiment of the integrated optoelectronic system was performed on the optical table in the laboratory to validate the performance of ARGO-M by using the laser system with high power and ultra-short pulse, ground target and receiving optics. It was demonstrated that PDU made by KASI provides the proper performance with RMS difference less than 0.4 ps in terms of system delay. According to the experiment results, the ranging accuracy of ARGO-M can be dozens of picoseconds. It was also shown that ARGO-M is capable of $2 \mathrm{kHz}$ laser ranging and that all components of the optoelectronic system can work properly.

\section{ACKNOWLEDGEMENTS}

This work was supported by the Korea Astronomy and Space Science Institute through the SLR system development program for space geodesy funded by the Ministry of Education Science and Technology (MEST).

\section{REFERENCES}

Bespalko V, Boole E, Vedin V, The model A032-ET of riga event timers, in Proceedings of the 15th International Workshop on Laser Ranging, Canberra, Australia, 15-20 Oct 2006, 321.

Iqbal F, Kirchner G, Koidl F, Fast response, medium resolution digital event timer and range gate generator for satellite laser ranging, ArtSa, 43, 143-149 (2008). doi: 10.2478/v10018-009-0013-8

Kirchner G, 2011, private communication.

Kirchner G, Koidl F, Short distance calibration, in Proceedings of the 10th International Workshop on Laser Ranging Instrumentation, Shanghai, China, 11-15 Nov 1996, 431.

Kirchner G, Koidl F, Prochazka I, Hamal K, Spad time walk compensation and return energy dependent ranging, in Proceedings of the 11th International Workshop on Laser Ranging, Deggendorf, Germany, 21-25 Sep 1998, 521.

Lim HC, Seo YK, Na JK, Bang SC, Lee JY, et al., Tracking capability analysis of ARGO-M satellite laser ranging system for STSAT-2 and KOMPSAT-5, JASS, 27, 245-252 (2010). doi: 10.5140/JASS.2010.27.3.245

Noll C, Pearlman M, International laser ranging service 2007-2008 report (Goddard Space Flight Center, Greenbelt, 2009), NASA/TP-2009-215848. 